\title{
Decreased Heart Rate Variability in Individuals With Amyotrophic Lateral Sclerosis
}

\author{
Renata M M Pimentel, Hugo Macedo Jr, Vítor E Valenti, Fernando O Rocha, Luiz Carlos Abreu, \\ Carlos Bandeira de M Monteiro, and Celso Ferreira
}

\begin{abstract}
BACKGROUND: Amyotrophic lateral sclerosis (ALS) is considered a multisystem degenerative disease due to its autonomic dysfunction effects. Autonomic cardiac control disorders can be seen in ALS and influence the quality of life and the life expectancy of affected individuals. We evaluated heart rate variability in subjects with ALS and with variable lung capacity. METHODS: We conducted a prospective cross-sectional study performed in 42 subjects with ALS. The subjects were classified into 2 groups according to their FVC: (FVC $>50 \%$ of the predicted value $[n=19]$ and FVC $<50 \%$ of the predicted value $[n=23])$. Heart rate was recorded at rest during spontaneous breathing by using a heart rate monitor. Linear indices in the time and frequency domains were analyzed, and non-linear analysis was performed by using Poincaré plots. RESULTS: The results showed a decrease of heart rate variability in the subjects with lower lung capacity and who needed ventilatory support. Qualitative analysis when using the plots supported the quantitative analysis, wherein the group with a lower lung capacity showed reduced heart rate variability. No significant differences were found in the other heart rate variability indices. CONCLUSIONS: The subjects with ALS and with decreased pulmonary capacity had reduced heart rate variability. Key words: motor neuron disease; amyotrophic lateral sclerosis; spirometry; autonomic nervous system; nervous system diseases; respiratory function. [Respir Care 2019;64(9):1088-1095. (C) 2019 Daedalus Enterprises]
\end{abstract}

\section{Introduction}

Amyotrophic lateral sclerosis (ALS) is a neurodegenerative disease characterized by the death of motor neurons, ${ }^{1}$ which eventually leads to respiratory failure and medulla oblongata dysfunction. ${ }^{2}$ There is recent evidence that an ALS diagnosis may be well established before the symptoms become apparent. ${ }^{3}$ According to the Brazilian Amyotrophic Lateral Sclerosis Association, ${ }^{3,4}$ a series of studies established that progressive bulbar palsy and pri-

Drs Renata M M Pimentel and Celso Ferreira are affiliated with the Programa de Pós Graduação em Medicina da Universidade Federal de São Paulo, Departamento de Cardiologia, São Paulo, Brazil. Drs Renata M M Pimentel, Hugo Macedo Jr, Fernando O Rocha, Luiz Carlos Abreu, and Carlos Bandeira de M Monteiro are affiliated with Laboratório de Delineamento da Escrita Científica, Faculdade de Medicina do ABC, Santo André, São Paulo, Brazil. Drs Hugo Macedo Jr and Luiz Carlos Abreu are affiliated with Programa de Pós Graduação em Ciências da Saúde da Coletividade da Faculdade de Medicina do ABC, Departamento de Saúde Coletiva, Santo Andre, São Paulo, Brazil. Dr Vítor E Valenti is affiliated with Programa de Pós-Graduação em Fisioterapia, UNESP, Presidente Prudente, São Paulo, Brazil. mary lateral sclerosis are subtypes of motor neuron disease. Motor neuron disease in general is related to a degenerative process of the central nervous system, with a variable clinical spectrum, but these subtypes could present different associated clinical characteristics. Twenty percent of patients begin in the bulbar group, which most often presents with either dysarthria or dysphagia. ${ }^{4}$

The association of motor neuron death with mutations in the $\mathrm{Cu}$ and $\mathrm{Zn}$ superoxide dismutase $(S O D 1)$ genes $^{5}$ is

\footnotetext{
This study received financial support from CNPq and Capes. English grammar and spelling was extensively reviewed by Hani K Atrash and by Proof-Reading Service, London, United Kingdom.
}

The authors have disclosed no conflicts of interest.

Correspondence: Renata M M Pimentel PhD, Laboratório de Delineamento da Escrita Científica, Faculdade de Medicina do ABC, Av. Lauro Gomes, 2000 Vila Sacadura Cabral, Santo André, SP, Brazil 09060-870. E-mail: re_pimentel1@ @otmal.com.

DOI: $10.4187 /$ respcare.06681 
in agreement with studies that demonstrated autonomic failure in subjects with ALS with a mutated SOD1 gene in neurons. ${ }^{6}$ Recent studies showed the existence of subclinical cardiovascular, gastrointestinal, sweating, and salivary and lacrimal dysfunctions, even in the early phases of ALS, which indicates abnormal sympathetic activity during the early stages of the disease with hyperhidrosis and a reduction in sweat production as the disease progresses. Furthermore, in advanced stages and in patients who are dependent on ventilatory support, apparent autonomic disorders may lead to circulatory collapse or sudden death. ${ }^{7,8}$ Thus, ALS is a degenerative multisystemic disease that results from related autonomic dysfunction.

Breathing modulates the autonomic flow to the heart and contributes to changes in heart rate variability. ${ }^{9,10} \mathrm{Be}-$ cause of the influence of breathing on the autonomic system, some studies have assessed heart rate variability while performing techniques used for respiratory therapy by integrating the management of cardiac care subjects. ${ }^{11}$ It is important to detect autonomic cardiac control disorders early because of the influence on survival and quality of life. ${ }^{12}$ Orthostatic hypotension, nocturnal blood pressure fluctuations, or falls and postural dizziness are increasingly reported. Studies reported decreased heart rate variability coincident with impaired salivary excretory function and gastrointestinal tract function, which indicate parasympathetic abnormalities, ${ }^{13,14}$ whereas other studies ${ }^{15}$ showed impaired cardiac sympathetic innervation. We believe that these associated disorders in different neurodegenerative diseases could affect the clinical course of ALS by increasing the risk of cardiac arrhythmia and sudden death.

The autonomic nervous system regulates the cardiovascular and respiratory systems. ${ }^{16}$ However, there is little understanding of how ALS is involved in cardiac arrhythmias. Because patients with ALS often have predominantly respiratory symptoms, diagnosis and treatment of cardiac arrhythmias can be difficult. ${ }^{17}$ Moreover, because the clinical course is dependent on disease progression and worsening of muscle and respiratory deficiencies, it is necessary to examine autonomic cardiac control in patients with motor neuron disease and to associate these findings with respiratory capacity. Therefore, we aimed to evaluate heart rate variability in ALS with and without bulbar palsy in subjects with variable lung capacity.

\section{Methods}

\section{STROBE Guidelines}

Our study conformed to the STROBE (Standards of Reporting of Observational Studies in Epidemiology) guidelines. Our investigation contained details of the study design, setting, participants, variables, data sources, mea-

\section{QUICK LOOK}

\section{Current knowledge}

Amyotrophic lateral sclerosis (ALS) is a multisystem degenerative disease, which can lead to several subclinical disorders linked to autonomic dysfunction. Changes in the autonomic nervous system are associated with cardiovascular dysfunctions. Because patients with ALS usually have predominantly respiratory symptoms, diagnosis and treatment of cardiac arrhythmias can be difficult.

\section{What this paper contributes to our knowledge}

Subjects with ALS with decreased lung capacity also had reduced heart rate variability. We believe that this autonomic dysfunction may affect the clinical course of ALS and increase the risk of cardiac arrhythmia and sudden death.

surement, description of potential sources of bias, quantitative variables description, and statistical methods.

\section{Population Study and Eligibility Criteria}

This study was performed in 42 subjects diagnosed with ALS. The group of subjects was divided and classified according to their FVC. The protocol was applied during the assessment carried out during subject visits. Subjects with an FVC $<50 \%$ of the predicted were using noninvasive ventilation, usually at night. No subject had used invasive mechanical ventilation. As inclusion criteria to participate in this study, we considered all the patients in clinical follow-up at the Department of Neuromuscular Diseases at Department of Neuromuscular Diseases of the Federal University of São Paulo diagnosed with ALS and with the confirmation of characteristics in El Escorial supported by an electroneuromyographic evaluation. ${ }^{18}$ All the participants provided signed informed consent (subject or representative). Patients with congenital abnormalities, heart disease, and pulmonary malformations, and those patients using drugs such as antiarrhythmics, insulin, and medications that influence the autonomic nervous system were excluded.

\section{Ethics Statement}

The study was approved by the ethics committee in Research of the Federal University of São Paulo (CAAE 30799414.9.0000.5505). 


\section{Decreased Heart Rate Variability in Subjects With ALS}

\section{Study Design and Setting}

This was a prospective, observational, and cross-sectional study. Recruitment was carried out by the Brazilian Amyotrophic Lateral Sclerosis Association in connection with the Department of Neuromuscular Diseases of the Federal University of São Paulo.

\section{Bias}

We performed all protocols by using the same conditions to address potential sources of bias. Variables were collected under controlled temperature $\left(21-25^{\circ} \mathrm{C}\right)$ and humidity (50-60\%). Abstinence from caffeine, alcohol, cola beverages, chocolate (for $12 \mathrm{~h}$ ), and exhaustive effort ( $24 \mathrm{~h}$ ) was recommended to individuals. Heart rate variability was evaluated between 1:00 PM and 6:00 PM to standardize the interference of circadian rhythm. All procedures necessary for data collection were explained to the individuals, and the subjects were instructed to remain at rest during the data collection.

\section{Initial Assessment and Experimental Protocols}

Data collection started after obtaining informed consent from the subjects or their representatives. Heart rate was recorded for 20 min while the subject was seated at rest and spontaneously breathing. Subsequently, spirometry was performed while the subject remained seated by the same respiratory therapist through a portable spirometer (MIR Spirobank version 3.6 New Berlin, WI) coupled to a microcomputer that used the Ocean \& WinSpiro software (New Berlin, WI) for Windows version 1.04 for analysis. The subjects were classified according to their FVC $(>50 \%$ or $<50 \%$ of the predicted value). Heart rate, breathing frequency, and oxygen saturation $\left(\mathrm{Sp}_{\mathrm{O}_{2}}\right)$ were also monitored at rest.

\section{Variables and Data Sources}

Instantaneous RR intervals were recorded with a digital telemetry system, which consisted of a transmitter placed on the subject's chest and a heart rate monitor. This equipment was previously validated to capture the heart rate for heart rate variability analysis. ${ }^{19}$ Oxygen saturation was determined by pulse oximetry (DX2010, Dixtal, São Paulo, SP, Brazil) connected to the subject's second finger through an appropriately sized sensor. The saturation of hemoglobin oxygen was recorded after stabilization as the most constant value during the second minute.

After placing the heart rate monitor, the participants remained seated, at rest. This position was chosen because many of the subjects were in a wheelchair throughout the day. In addition, most patients do not remain in the supine position due to diaphragmatic muscle weakness, which may result in discomfort and cause a significant change in heart rate variability. We respected the initial 5-min rest interval to stabilize the heart rate. For analyzing heart rate variability data, we selected consecutive stable RR intervals, then filtering was performed through Microsoft Excel (Microsoft, Redmond, WA) to eliminate artifacts. The first 1,000 intervals of beats were chosen, and only series with $>95 \%$ sinus beats were included in the study. ${ }^{20}$ The software used for heart rate variability evaluation was Kubios heart rate variability version 2.0 (Helsinki, Finland).

\section{Outcome Measures}

We analyzed the time and frequency domain heart rate variability indices and non-linear methods through Poincaré plots. For heart rate variability analysis in the frequency domain, we used spectral components of low frequency $(0.04-0.15 \mathrm{~Hz})$ and high frequency $(0.15-0.40 \mathrm{~Hz})$ in $\mathrm{ms}^{2}$ and normalized units (nu). Spectral analysis was calculated by using the fast Fourier transform algorithm. The analysis in the time domain was performed through the mean \pm SD of normal RR intervals) and the root of the square successive differences between adjacent normal RR intervals. We used the Kubios heart rate variability analysis software for the linear analysis of heart rate variability. ${ }^{21}$

The RR triangular index was calculated based on the construction of the density histogram of normal RR intervals and was obtained by dividing the histogram integral interval (ie, the total number of RR intervals) by the maximum density distribution (modal frequency of RR intervals) measured on a discrete scale with boxes of $7.8125 \mathrm{~ms}$ $(1 / 128 \mathrm{~s}) .{ }^{22}$ The triangular interpolation of RR intervals constitutes the width of the baseline distribution as measured by the base of a triangle; the distribution of all RR intervals and the difference of least squares were used to determine the triangle. ${ }^{22}$

The heart rate variability Poincaré plot was analyzed from the RR intervals based on non-linear dynamics. ${ }^{23,24}$ This analysis was a 2-dimensional graphic representation of the correlation between consecutive RR intervals, in which each interval was plotted against the next interval. ${ }^{23,25,26}$ For the quantitative analysis of the Poincaré plots, the following indices were calculated: SD of the instantaneous variability beat by beat (SD1), SD for longterm of continuous RR intervals (SD2), and SD1/SD2. ${ }^{27}$ Qualitative analysis (visual) of the Poincaré plots was performed through the analysis of the figures formed by the attractor of the plot, which were described by Tulppo et al ${ }^{28}$ :

1. A figure in which an increase in the dispersion of $R R$ intervals beat by beat is observed, with an increase in RR intervals, characteristic of a normal plot. 


\section{Decreased Heart Rate Variability in Subjects With ALS}

Table 1. Distribution of Study Subjects by Age, Sex, Baseline Heart Rate, Oxygen Saturation

\begin{tabular}{lcccc}
\hline \hline \multicolumn{1}{c}{ Variable } & FVC $>50 \%$ & FVC $<50 \%$ & $P$ & Cohen's d \\
\hline Age, mean \pm SD y & $56.5 \pm 9.8$ & $58.7 \pm 9.2$ & .47 & NA \\
Males, $n$ & 8 & 12 & NA & NA \\
Females, $n$ & 11 & 11 & NA & NA \\
Heart rate, mean \pm SD beats/min & $80.11 \pm 10.03$ & $87.3 \pm 10.44$ & .03 & .008 \\
Oxygen saturation, mean \pm SD $\%$ & $96.41 \pm 2.12$ & $94 \pm 3.1$ & 0.9
\end{tabular}

$\mathrm{NA}=$ not applicable

2. A figure with little global dispersion beat by beat and without increased dispersion of RR intervals in the long term.

Kubios Heart Rate Variability software Version 2.0 was used to assess these indices. ${ }^{21}$ Vital capacity was measured by spirometry. The subjects were classified into 2 groups according to their lung capacity (FVC). Individuals with ALS were distributed into the spirometry group $<50 \%$ $($ FVC $<50 \%$ predicted $[n=23])$ and the spirometry group $>50 \%$ (FVC $>50 \%$ predicted $[n=19])$. Based on these parameters, the subjects with $\mathrm{FVC}<50 \%$ predicted required ventilatory support. In neuromuscular diseases, for example, ALS, an FVC $<50 \%$ of the predicted value is an indication for ventilatory support with 2 pressure levels, even in the absence of respiratory symptoms. ${ }^{29-32}$

\section{Study Size}

To determine the sample size, previous knowledge was required based on the mean $\pm \mathrm{SD}$ of normal $\mathrm{RR}$ intervals index in a study by Moreno et al. ${ }^{33} \mathrm{~A}$ sample size of 18 participants per group was stipulated by a hypothesis test (2-tailed), with a 5\% level of significance and $80 \%$ power.

\section{Statistical Analysis}

Qualitative variables were analyzed by using the Fisher exact test or the chi-square test. The data are presented as box plots. We used the Shapiro-Wilk normality test to evaluate the distribution. For parametric distributions, we applied the unpaired Student $t$ test; whereas, for nonparametric distributions, we applied the MannWhitney test. All findings with $P<.05$ were considered statistically significant. To measure the magnitude of the difference between the groups, the effect size was calculated by using Cohen's d for significant differences. A large effect was considered for values $>0.9$, moderate for values between 0.9 and 0.5 , and small for values between 0.5 and 0.25 .
Table 2. Frequency Domain Indices

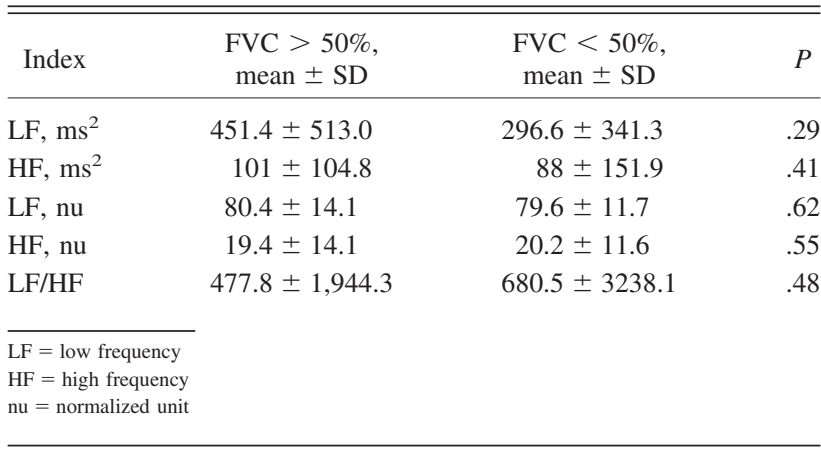

\section{Results}

\section{Population Characteristics}

Forty-two subjects diagnosed with motor neuron disease were evaluated: $47.5 \%$ were female and $52.5 \%$ were male subjects. The mean age was $57.7 \mathrm{y}$. The subjects had been diagnosed with motor neuron disease, on average, 3 y earlier, and 17 subjects had bulbar disease characteristics. Data regarding age, sex, baseline heart rate, and oxygen saturation of the groups are shown in Table 1. We noted that the resting heart rate was higher, whereas $\mathrm{S}_{\mathrm{pO}_{2}}$ was lower in the $\mathrm{FVC}<50 \%$ group. In relation to the lung capacity of the 2 groups, the $\mathrm{FVC}<50 \%$ group was mean $\pm \mathrm{SD}, 38.06 \pm 0.10 \%$, whereas the $\mathrm{FVC}>50 \%$ group was mean $\pm \mathrm{SD}, 73.54 \pm 0.097 \%$.

\section{Linear Methods}

We found no significant differences between the groups in the frequency domain analysis (Table 2).

\section{Geometric Heart Rate Variability Indices}

With regard to the geometric indices, we observed increased SD2 in the FVC $>50 \%$ group (moderate effect size) (Table 3). Shown in Figure 1, is an example of a Poincaré plot from one subject in the FVC $>50 \%$ group 


\section{Decreased Heart Rate Variability in Subjects With ALS}

Table 3. Geometric Indices of Heart Rate Variability

\begin{tabular}{lcccc}
\hline \hline Index & $\begin{array}{c}\text { FVC }>50 \%, \\
\text { mean } \pm \text { SD }\end{array}$ & $\begin{array}{c}\text { FVC }<50 \%, \\
\text { mean } \pm \text { SD }\end{array}$ & $P$ & Cohen's d \\
\hline RRtri & $11.28 \pm 6.01$ & $8.7 \pm 4.6$ & .08 & ND \\
TINN & $186.17 \pm 84.03$ & $144.13 \pm 81.31$ & .14 & ND \\
SD1 & $11.4 \pm 6.0$ & $9.9 \pm 8.3$ & .15 & ND \\
SD2 & $56.0 \pm 22.6$ & $38.6 \pm 18.9$ & .01 & 0.83 \\
& & & \\
RRtri $=$ triangular index & & & \\
ND $=$ no data \\
TINN = triangular interpolation of RR intervals \\
SD1 = SD of instantaneous beat-by-beat variability \\
SD2 = long-term SD of continuous RR intervals \\
\end{tabular}

(Fig. 1A) and one subject in the FVC $<50 \%$ group (Fig. 1B). We noted less dispersion in the points of subjects with reduced lung capacity. We performed a correlation analysis to verify whether there is an association between respiratory function and autonomic control of heart beats in both groups (Table 4). There was no significant correlation between FVC and heart rate variability in both groups.

\section{Discussion}

Our main finding indicated a significant decrease $(P<0.01)$ in the SD2 index in the subjects who needed ventilatory support, which indicated reduced overall heart rate variability modulation. We also found a higher heart rate in the FVC $<50 \%$ group, which reinforced the impaired autonomic function; whereas $\mathrm{S}_{\mathrm{pO}_{2}}$ was decreased in the same group, which supported the worst pulmonary condition in this group. In this sense, our findings supported that breathing modulates autonomic flow and contributed to changes in heart rate variability. ${ }^{10}$

Spirometric measurements showed that, in most cases, regardless of motor neuron failure, FVC decreased with progression of the disease. ${ }^{34}$ The restrictive lung pattern in ALS is characterized by an increased residual volume with reduced total lung capacity. ${ }^{35}$ Moreover, inspiratory and expiratory pressures are also reduced, and pressure decreases according to the disease evolution. Previous studies reported that dyspnea is generally associated with diaphragmatic dysfunction, respiratory pulse, and a delay in or abolition of diaphragm responses to stimulation of the phrenic nerve or cortical stimulation. ${ }^{34,36}$ These results were consistent with previous observations of predominantly phrenic nerve neuropathologic abnormalities in subjects with ALS. 34,36

The presence of $\mathrm{FVC}<50 \%$ predicted in motor neuron disease requires bi-level ventilatory support, even in the absence of respiratory symptoms. ${ }^{30,32}$ Verification of heart rate variability is important to detect autonomic dysfunction disorders in patients with motor neuron disease to avoid sudden death or other conditions that lead to a decrease in life expectancy. ${ }^{12}$ Autonomic dysfunction also supports the concept that ALS is a degenerative disease of multiple systems. ${ }^{6}$

Autonomic dysfunction (hyperhidrosis, alterations in salivary and gastrointestinal tract function) usually leads to subclinical symptoms. Patients in more-advanced stages of disease that requires ventilatory support often show autonomic nervous system disorders. Such responses are marked by fluctuations in blood pressure and heart rate, and can occur in clinically stable patients with ventilatory support. This response is known as the autonomic storm and is characterized by a hypertensive crisis with systolic blood pressure $>250 \mathrm{~mm} \mathrm{Hg}$ and tachycardia. ${ }^{6}$ These autonomic symptoms are similar to baroreflex failure; they indicate a poor prognosis and may result in sudden death. ${ }^{6}$ In this situation, careful assessment and individual treatment are strongly indicated, although adequate therapeutic approaches have not been well established. Autonomic dysfunction may be clinically obvious and devastating, especially at an advanced stage when ventilatory support is required.

Our results showed no significant correlation between heart rate variability and pulmonary capacity, which is supported by previous studies (Table 4). ${ }^{15,37}$ We hypothesize that the absence of significant differences in the time (mean \pm SD of normal RR intervals, percentage of adjacent RR intervals with a difference of duration of $>50 \mathrm{~ms}$, and the root of the square successive differences between adjacent normal RR intervals) and frequency (high frequency, low frequency, and low frequency to high frequency ratio) domains is because geometric indices are more sensitive at detecting changes. In this sense, nonlinear behavior is predominant in human systems due to their complex and dynamic nature. Human physiology cannot be accurately described by linear methods. Chaos theory describes elements that manifest behaviors that are extremely sensitive to initial conditions but that are difficult to repeat and are deterministic. ${ }^{20}$

The analysis of non-linear heart rate variability methods has been gaining increasing interest, because there is evidence that the mechanisms involved in cardiovascular regulation probably interact in a non-linear way. This new insight into abnormalities in heart rate behavior under various conditions may provide additional prognostic information when compared with traditional methods. However, its clinical utility requires further research with large populations. ${ }^{38}$

Heart rate variability was analyzed from the RR intervals through Poincaré plots based on non-linear dynamics. ${ }^{23,24}$ The Poincare plot is a 2-dimensional graphic representation of the correlation between consecutive RR intervals in which each interval is plotted against the next interval. ${ }^{23,25,26}$ The Poincaré plot can be analyzed qualita- 

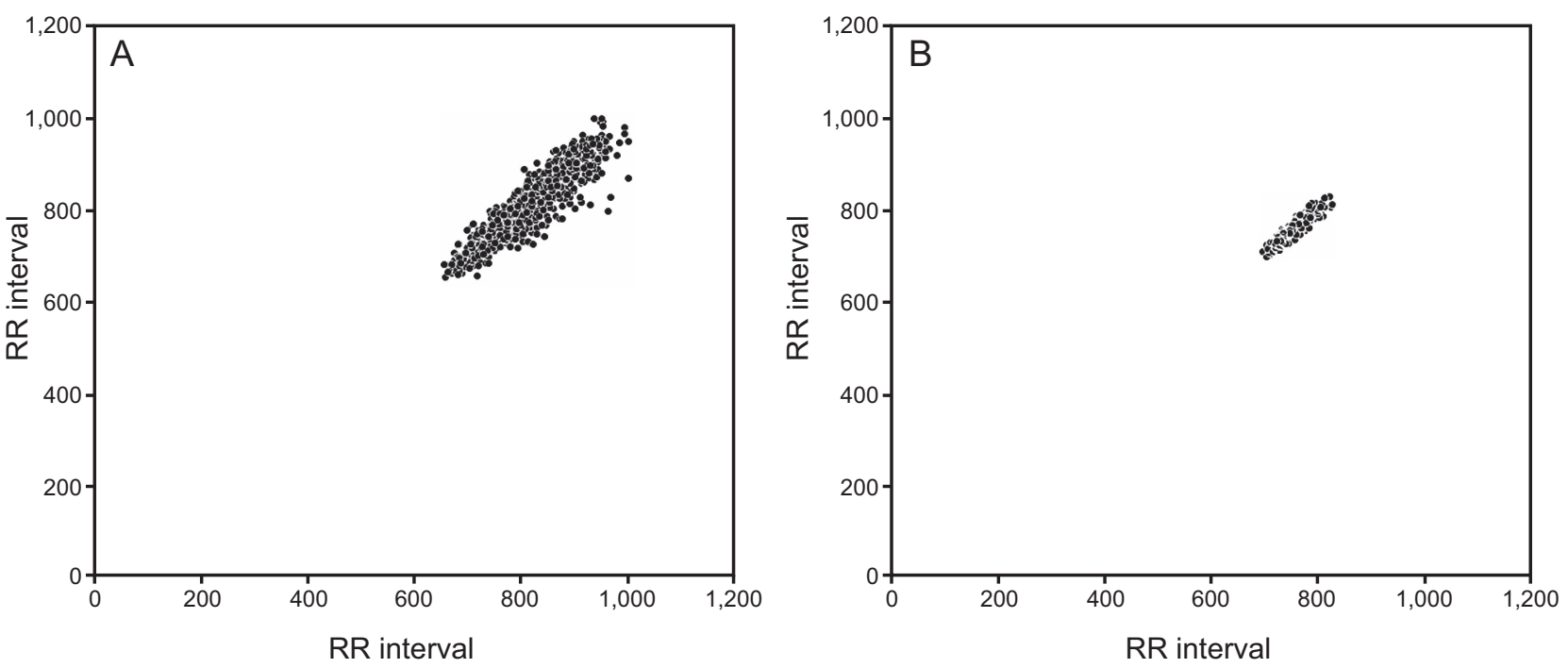

Fig. 1. Visual pattern of the Poincaré plot observed in one subject with FVC $>50 \%(A)$ and another with FVC $<50 \%$ (B).

Table 4. Correlation Between FVC and Heart Rate Variability in the Both Groups

\begin{tabular}{|c|c|c|c|c|}
\hline \multirow{2}{*}{ Parameter } & \multicolumn{2}{|c|}{$\mathrm{FVC}>50 \%$} & \multicolumn{2}{|c|}{$\mathrm{FVC}<50 \%$} \\
\hline & $\mathrm{r}$ & $P$ & $\mathrm{r}$ & $P$ \\
\hline $\mathrm{RR}$ interval & 0.44 & .058 & -0.24 & .26 \\
\hline SDNN & -0.34 & .15 & -0.31 & .15 \\
\hline Heart rate & -0.37 & .11 & 0.18 & .40 \\
\hline RMSSD & -0.06 & .80 & -0.21 & .33 \\
\hline pNN50 & -0.1 & .66 & -0.1 & .64 \\
\hline RRtri & -0.26 & .27 & -0.29 & .17 \\
\hline TINN & -0.33 & .16 & -0.24 & .25 \\
\hline $\mathrm{LF}, \mathrm{ms}^{2}$ & -0.23 & .34 & -0.33 & .11 \\
\hline LF, nu) & -0.18 & .44 & -0.05 & .81 \\
\hline $\mathrm{HF}, \mathrm{ms}^{2}$ & -0.15 & .53 & -0.14 & .49 \\
\hline $\mathrm{HF}, \mathrm{nu}$ & 0.18 & .44 & 0.05 & .81 \\
\hline $\mathrm{LF} / \mathrm{HF}$ & -0.06 & .80 & -0.15 & .48 \\
\hline SD1 & -0.06 & .80 & -0.2 & .34 \\
\hline $\mathrm{SD} 2$ & -0.39 & .09 & -0.22 & .30 \\
\hline
\end{tabular}

$\overline{\mathrm{SDNN}}=\mathrm{SD}$ of all NN $\mathrm{intervals}$

RMSSD $=$ square root of the mean of the sum of the squares of differences between adjacent $\mathrm{NN}$ intervals

pNN50 = percentage of adjacent RR intervals with a difference of duration of $>50 \mathrm{~ms}$ RRtri $=$ triangular index

TINN $=$ triangular interpolation of RR intervals

$\mathrm{LF}=$ low frequency

$\mathrm{HF}=$ high frequency

$\mathrm{nu}=$ normalized units

$\mathrm{SD} 1=\mathrm{SD}$ of instantaneous beat-by-beat variability

$\mathrm{SD} 2$ = long-term SD of continuous RR intervals

tively by evaluating the shape formed by its attractor, which shows the degree of RR interval complexity, ${ }^{39}$ as the results show in Figure 1, or it can be analyzed quantitatively (as shown in Table 2) by setting an ellipse to the shape formed by the plot, in which the SD1 and SD2 indices are generated. ${ }^{27}$ These methods describe the complex fluctu- ations of the rhythm and can separate structures of nonlinear behavior in a temporal series of heartbeats more adequately than linear methods. ${ }^{36}$ It has been speculated that heart rate variability analysis based on non-linear, dynamic methods can provide valuable information for physiologic interpretations of heart rate variability ${ }^{40}$ and assess the risk of sudden death. ${ }^{8,24}$

Pinto et $\mathrm{al}^{8}$ reported that a low coefficient of variation of heart rate is a potential prognostic marker in ALS and other forms of motor neuron disease, and may lead to sudden death. Our results indicated a decrease in SD2 in subjects with greater respiratory impairment (heart rate variability $<50 \%$ ), which required ventilatory support. The SD2 index represents both the vagal and sympathetic modulation of HR. ${ }^{41}$ Reduced SD2 was suggested to be an indicator of cardiac mortality and morbidity. ${ }^{42}$ Taken together, we reinforced that declined SD2 in patients with ALS and with lower pulmonary capacity was related to a worse prognosis.

For visual, qualitative, analysis of the Poincaré plot, it is clear that the group with FVC $<50 \%$ predicted presented a more-flattened form with less dispersion of RR intervals, which indicated decreased heart rate variability. In this sense, the plot supported the quantitative analysis of SD2, which showed reduced values and worse autonomic function in the ALS group with FVC $<50 \%$. The theories of non-linear systems have been progressively applied to interpret, explain, and predict the behavior of biological phenomena. These parameters have been found to be good predictors of morbidity and mortality in the clinical context, despite the need for deeper scientific analysis, with expressive samples and prolonged follow-up. Such studies may be useful in research and treatment of heart disease. ${ }^{43}$ 


\section{Decreased Heart Rate Variability in Subjects With ALS}

Disturbances in cardiac autonomic control in patients with ALS with and without progressive bulbar palsy can influence survival and quality of life because this is associated with the onset of a hypertensive crisis, sudden cardiac death, and cardiovascular collapse, all leading to a decrease in life expectancy. ${ }^{13,14,44}$ Also worth mentioning is that the associations tested through Pearson and Spearman procedures were not statistically significant. We believe that patients with ALS and with lower FVC are influenced by physiologic variables not evaluated, including cytokines, ${ }^{45}$ antioxidant status, ${ }^{46}$ and $\alpha$-amylase.

This study showed that heart rate variability may help to provide information regarding the lung function of patients with ALS. However, because lung function was measured only by spirometry, our results should be considered with caution. Heart rate variability changes induced by autonomic change could provide important data about the interaction between heart rate autonomic control and FVC in patients with ALS. Thus, we suggest that further studies of this procedure are needed. A limitation of the present study is that we did not monitor breathing pattern and tidal volume during heart rate recording. The above-mentioned variables mainly influence the high frequency band. ${ }^{22}$ During heart rate variability analysis, all the subjects were breathing spontaneously.

\section{Conclusions}

Our study showed that subjects with ALS and with reduced lung capacity presented impaired autonomic regulation of the heart rate. We suggest that impaired lung capacity was associated with autonomic dysfunction in subjects with ALS. This finding is important for the clinical team to plan therapies and treatment based on patients' lung and autonomic function.

\section{ACKNOWLEDGMENTS}

We thank the reviewers for the anonymous constructive review on our manuscript and for providing interesting ideas for future projects.

\section{REFERENCES}

1. Hayashi Y, Homma K, Ichijo H. SOD1 in neurotoxicity and its controversial roles in SOD1 mutation-negative ALS. Adv Biol Regul 2016;60:95-104.

2. Baydur A. Respiratory muscle strength: a reliable index for predicting survival in amyotrophic lateral sclerosis? Am J Respir Crit Care Med 2017;195(1):12-13.

3. Menke RA, Proudfoot M, Wuu J, Andersen PM, Talbot K, Benatar $\mathrm{M}$, Turner MR. Increased functional connectivity common to symptomatic amyotrophic lateral sclerosis and those at genetic risk. J Neurol Neurosurg Psychiatry 2016;87(6):580-588.

4. Elman LB, McCluskey L. Clinical features of amyotrophic lateral sclerosis and other forms of motor neuron disease. Up-to-date. Waltham: Wolters Kluwer Health; 2012.
5. Proctor EA, Fee L, Tao Y, Redler RL, Fay JM, Zhang Y, et al. Nonnative SOD1 trimer is toxic to motor neurons in a model of amyotrophic lateral sclerosis. Proc Natl Acad Sci U S A 2016;3(3): 614-619.

6. Kékesi O, Bellot-Saez A Seah W, Berg T, Trpceski M, Yerbury JJ, Ooi L. Dynamic interplay between H-current and M-current controls motoneuron hyperexcitability in amyotrophic lateral sclerosis. Cell Death Dis. 2019;10(4):310.

7. Baltadzhieva R, Gurevich T, Korczyn AD. Autonomic impairment in amyotrophic lateral sclerosis. Curr Opin Neurol 2005;18(5):487-493.

8. Pinto S, Pinto I, De Carvalho M. Decreased heart rate variability predicts death in amyotrophic lateral sclerosis. Muscle Nerve 2012; 46(3):341-345.

9. Bernardi L, Porta C, Gabutti A, Spicuzza L, Sleight P. Modulatory effects of respiration. Auton Neurosci 2001;90(1-2):47-56.

10. Bernardi L, Gabutti A, Porta C, Spicuzza L. Slow breathing reduces chemoreflex response to hypoxia and hypercapnia, and increases baroreflex sensitivity. J Hypertens 2001;19(12):2221-2229.

11. Lacerda FA, Garro SA, Aguiar SG, Pessoa BP, Figueiredo PHS, Diniz GC. Heart rate variability during the use of incentive spirometers. Rev Bras Cardiol 2014;27(3):158-164.

12. Martínez J, Ramón C, Morís C, Pascual J, Morís G. Abnormal electrocardiogram in a subject with amyotrophic lateral sclerosis mimicking myocardial ischaemia. World J Clin Cases 2014;2(6):211214.

13. Shimizu T, Hayashi H, Kato S, Tanabe H. Hyposensitivy of peripheral alpha-adrenoceptors in respiratory-dependent amyotrophic lateral sclerosis assessed by intravenous norepinephrine infusion. Clin Auton Res 1995;5(3):165-169.

14. Pavlovic S, Stevic Z, Milovanovic B. Impairment of cardiac autonomic control in subjects with amyotrophic lateral sclerosis. Amyotroph Lateral Scler 2010;11(3):272-276.

15. Merico A, Cavinato M. Autonomic dysfunction in the early stage of ALS with bulbar involvement. Amyotroph Lateral Scler 2011;12(5): 363-367.

16. Pinheiro CH, Medeiros RA, Pinheiro DG, Marinho Mde J. Modification of the respiratory pattern improves cardiovascular control in essential hypertension. Arq Bras Cardiol 2007;88(6):651-659.

17. Shemisa K, Kaelber, D, Parikh SA, Mackall JA. Autonomic etiology of heart block in ALS: case report. J Med Case Rep 2014;8:224.

18. Brooks BR. El Escorial World Federation of Neurology criteria for the diagnosis of amyotrophic lateral sclerosis. Subcommittee on Motor Neuron Diseases/Amyotrophic Lateral Sclerosis of the World Federation of Neurology Research Group on Neuromuscular Diseases and the El Escorial "Clinical limits of amyotrophic lateral sclerosis" workshop contributors. J Neurol Sci 1994;124(Suppl):96107.

19. Williams DP, Jarczok MN, Ellis RJ, Hillecke TK, Thayer JF, Koenig J. Two week test-retest reliability of the Polar ${ }^{\circledR} \mathrm{RS} 800 \mathrm{CX}^{\mathrm{TM}}$ to record heart rate variability. Clin Physiol Funct Imaging 2017;37(6): 776-781.

20. Sassi R, Cerutti S, Lombardi F, Malik M, Huikuri HV, Peng CK, et al. Advances in heart rate variability signal analysis: joint position statement by the e-Cardiology ESC Working Group and the European Heart Rhythm Association co-endorsed by the Asia Pacific Heart Rhythm Society. Europace. 2015;17(9):1341-1353.

21. Niskanen JP, Tarvainen MP, Ranta-Aho PO, Karjalainen PA. Software for advanced HRV analysis. Comput Methods Programs Biomed 2004;76(1):73-81.

22. Task Force of the European Society of Cardiology and the North American Society of Pacing and Electrophysiology. Heart rate variability. Standards of measurement, physiologic interpretation, and clinical use. Circulation 1996;93(5):1043-1065. 


\section{Decreased Heart Rate Variability in Subjects With ALS}

23. Khaled AS, Owis Mi, Mohamed Asa. Employing time-domain methods and poincaré plot of heart rate variability signals to detect congestive heart failure. BIME J 2006;6(1):35-41. Available at: https:// www.researchgate.net/profile/Mohamed_Owis2/publication/253138 855_Employing_Time-Domain_Methods_and_Poincare_Plot_of_ Heart_Rate_Variability_Signals_to_Detect_Congestive_Heart_Failure/ links/00b7d5265a63fa2cb2000000/Employing-Time-Domain-Methodsand-Poincare-Plot-of-Heart-Rate-Variability-Signals-to-Detect-Congest ive-Heart-Failure.pdf. Accessed July 20, 2018.

24. Voss A, Schroeder R, Truebner S, Goernig M, Figulla HR, Schirdewan A. Comparison of nonlinear methods symbolic dynamics, detrended fluctuation, and Poincare plot analysis in risk stratification in patients with dilated cardiomyopathy. Chaos 2007;17(1):015120.

25. Lerma C, Infante O, Pérez-Grovas H, José MV. Poincaré plot indexes of heart rate variability capture dynamic adaptations after haemodialysis in chronic renal failure subjects. Clin Physiol Funct Imaging 2003;23(2):72-80.

26. Smith AL, Reynolds KJ, Owen H. Correlated Poincaré indices for measuring heart rate variability. Australas Phys Eng Sci Med 2007; 30(4):336-341.

27. Dias de Carvalho T, Marcelo Pastre C, Claudino Rossi R, de Abreu LC, Valenti VE, Marques Vanderlei LC. Geometric index of heart rate variability in chronic obstructive pulmonary disease. Rev Port Pneumol. 2011;17(6):260-265.

28. Tulppo MP, Mäkikallio TH, Seppänen T, Laukkanen RT, Huikuri HV. Vagal modulation of heart rate during exercise: effects of age and physical fitness. Am J Physiol 1998;274(2):H424-429.

29. Prell T, Ringer TM, Wullenkord K, Garrison P, Gunkel A, Stubendorff $\mathrm{B}$, et al. Assessment of pulmonary function in amyotrophic lateral sclerosis: when can polygraphy help evaluate the need for non-invasive ventilation? J Neurol Neurosurg Psychiatry 2016;87(9): 1022-1026.

30. Ambrosino N, Carpenè N, Gherardi M. Chronic respiratory care for neuromuscular diseases in adults. Eur Respir J 2009;34(2):444-451.

31. Vrijsen B, Testelmans D, Belge C. Non-invasive ventilation in amyotrophic lateral sclerosis. Amyotroph Lateral Scler Frontotemporal Degener 2013;14(2):85-95.

32. Trindade AM, Sousa TLF, Albuquerque ALP. The interpretation of spirometry in pneumological practice: how far can we proceed with the use of its parameters? Pulmão RJ 2015;24(1):3-7. Available at: http://www.sopterj.com.br/wp-content/themes/_sopterj_redesign_ 2017/_revista/2015/n_01/04.pdf.

33. Moreno IL, Pastre CM, Ferreira C, de Abreu LC, Valenti VE, Vanderlei LC. Effects of an isotonic beverage on autonomic regulation during and after exercise. J Int Soc Sports Nutr 2013;10(1):2.
34. Arnulf I, Similowski T, Salachas F, Garma L, Mehiri S, Attali V, et al. Sleep disorders and diaphragmatic function in patients with amyotrophic lateral sclerosis. Am J Respir Crit Care Med 2000; 161(3 Pt 1):849-856.

35. Pais MF, Tyner T, Jain VV, Vempilly JJ. Characterizing respiratory muscle weakness in amyotrophic lateral sclerosis with the combined use of lung volumes, spirometry and muscle strength. Am J Respir Crit Care Med 2016;193(1):A6364. Available at: https://www.atsjournals.org/doi/ pdf/10.1164/ajrccm-conference.2016.193.1_MeetingAbstracts.A6364.

36. Georges M, Morélot-Panzini C, Similowski T, Gonzalez-Bermejo J. Noninvasive ventilation reduces energy expenditure in amyotrophic lateral sclerosis. BMC Pulm Med 2014;14:17. Accessed June 9, 2018.

37. Pisano F, Miscio G, Mazzuero G, Lanfranchi P, Colombo R, Pinelli $P$. Decreased heart rate variability in amyotrophic lateral sclerosis. Muscle Nerve 1995;18(11):1225-1231.

38. Huikuri HV, Mäkikallio TH, Perkiömäki J. Measurement of heart rate variability by methods based on nonlinear dynamics. J Electrocardiol 2003;36(Suppl):95-99.

39. De Vito G, Galloway SD, Nimmo MA, Maas P, McMurray JJ. Effects of central sympathetic inhibition on heart rate variability during steady-state exercise in healthy humans. Clin Physiol Funct Imaging 2002;22(1):32-38

40. Higgins JP. Nonlinear systems in medicine. Yale J Biol Med 2002; 75(5-6):247-260.

41. Carvalho TD, Pastre CM, de Godoy MF, Fereira C, Pitta FO, de Abreu LC, et al. Fractal correlation property of heart rate variability in chronic obstructive pulmonary disease. Int J Chron Obstruct Pulmon Dis 2011;6:23-28.

42. Manzano BM. Variability of heart rate as a tool to analyze the autonomic function of smokers: literature review and Poincaré plot study [master's dissertation]. Presidente Prudente UNESP: Presidente Prudente, Paulista State University; 2009.

43. Vanderlei LC, Pastre CM, Hoshi RA, Carvalho TD, Godoy MF. Basic notions of heart rate variability and its clinical applicability. Rev Bras Cir Cardiovasc 2009;24(2):205-217.

44. Gil J, Funalot B, Verschueren A. Causes of death amongst French subjects with amyotrophic lateral sclerosis: a 217 prospective study. Eur J Neurol 2008;15(11):1245-1251.

45. Ask TF, Lugo RG, Sütterlin S. The Neuro-Immuno-Senescence Integrative Model (NISIM) on the negative association between parasympathetic activity and cellular senescence. Front Neurosci 2018; 12:726.

46. Cipak Gasparovic A, Zarkovic N, Zarkovic K, Semen K, Kaminskyy D, Yelisyeyeva O, Bottari SP. Biomarkers of oxidative and nitrooxidative stress: conventional and novel approaches. Br J Pharmacol 2017;174(12):1771-1783. 